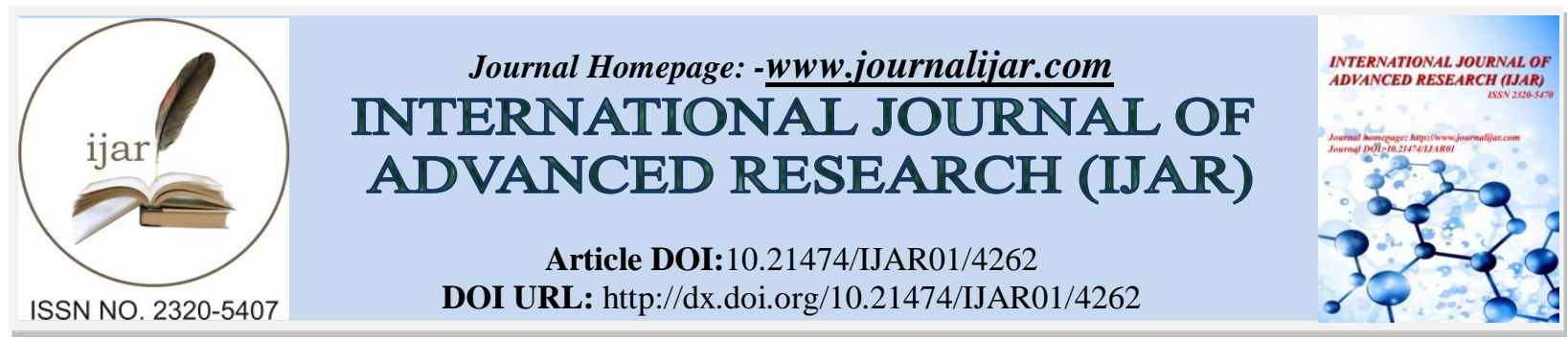

RESEARCH ARTICLE

\title{
POLITICAL CONFLICT MANAGEMENT IN THE GREAT LAKES REGION: RWANDA, BURUNDI AND DRCONGO.
}

Mukandirwa Salumu.

\section{Manuscript Info}

Manuscript History

Received: 27 March 2017

Final Accepted: 30 April 2017

Published: May 2017

Key words:-

Management, conflict, political,

management.

\section{Abstract}

The purpose of this study was to examine the Political Conflict Management in the Great Lakes Region, case of Rwanda, Burundi and DR Congo. The specific question studied in this research was to understand the political conflict management model adopted in Rwanda, Burundi and DR Congo in order to abolish all kinds of conflict.

The researcher adopted literature review and interview research design to collect information concerning the Political Conflict Management in the Great Lakes Region, case of Rwanda, Burundi and DR Congo and the model adopted by each country. Qualitative method was used and it is the most popular research method within the great lakes region realities.

The study was guided by hypothesis that there was a significant difference between Great Lakes countries model of political conflict management. The political instruments used for the Rwanda Government in order to rebuild the unit and the reconciliation of the Rwandan. The government was inspired by the reconstruction of a Rwandan nation, by the Rwandity. The political instrument used in Burundi to resolve the Burundian conflict is the ethnic institutionalization (ethnic quota: 40/100 for Tutsi and 60/100 for Hutu). The political instruments used in DR Congo to make the end of conflict was the global and inclusive agreement on the transition, be inspired by the reconstruction of the nation by the sharing of the power between the political parties (quota of the political parties).

It's concluded that the best model of political conflict management is for Rwanda Government because it does not make any differences among its citizen but it provides rwandity or merit system.

Copy Right, IJAR, 2017,. All rightsreserved.

\section{Introduction:-}

La partie introductive présente les grandes articulations de ce travail. Elle donne le contexte, l'hypothèse, la justification, l'objectif et la méthodologie.

Contexte, Hypothèse, Justification Et Objectifs:-

Lorsque quelques messieurs bien-pensant se mirent à dessiner les frontières de l'Afrique à Berlin en 1885, aucun d'eux n'y avait déjà mis les pieds, ne connaissaient aucune réalités, langues, peuples, culture, etc. 
Ils se sont donné l'audace de tailler à coup de crayons et compas des frontières qui correspondaient non aux réalités, mais uniquement à leurs intérêts. Cette Afrique qu'ils taillaient en pièce, était en réalité composée de grands et petits royaumes, ne correspondant en rien aux limites actuelles.

Le morcellement de l'Afrique n'avait pas épargné la région des grands lacs, c'est ainsi qu'une même famille se retrouvant de part et d'autres de la frontières, ses membres appartiennent à deux ou trois pays différents et ayant des langues officielles différentes.

On estime que dans les années nonante, l'Afrique a connu une prolifération de conflits armés, à mesure qu'un grand nombre de régimes militaires et de régimes a parti unique ont été déstabilisés par la fin de la guerre froide. La montée de mouvement démocratique et les conflits violents qui ont été provoqués par la discrimination, par l'exclusion économique, sociale et politique. Dans le pire des cas, l'intolérance extrême des gens à l'égard d'une identité ethnique différente s'est manifestée par des massacres et des actes de génocide.

C'est ainsi qu'en 1994 il y a eu la violence du génocide au Rwanda qui franchit les frontières pour se répandre au Burundi et au Congo, drainant des millions de réfugiés aussi que les reliquats des armées et des guérillas qui avaient conduit le génocide, groupe attisé par l'idéologie de la haine ethnique. Les conflits avaient déjà commencés en Congo, et au Burundi, mais le débordement du génocide les convertit en un supplice sans issue pour toute la région des grands lacs.

Face à cette situation problématique, nous nous sommes posé une question, qui est la suivante : Quels sont les modèles de gestion politique des conflits adoptés par le Rwanda, le Burundi et la RD Congo ?

La réponse anticipative à cette question est la reconstruction de la nation fondée sur l'unité nationale au Rwanda, au Burundi et en RD Congo serait l'un des moyens de résolution des conflits dans ces pays.

La présente étude s'intéresse à une analyse comparative et critique des initiatives régionales de paix et notamment de celles qui ont apporté une contribution jugée significative au processus de rétablissement de la paix dans la région des grands lacs.

L'objectif de cette étude est d'enrichir la connaissance des pratiques régionales de gestion des conflits, et d'aider à mieux appréhender en quoi et comment ont-elles contribuent à la paix dans la région.

Tableau Synthétique Des Méthodes Utilisées

\begin{tabular}{|l|l|l|}
\hline \multicolumn{2}{|c|}{ Etape de la recherche } & \multicolumn{1}{c|}{ Méthodeou technique } \\
\hline 1. & La récolte des données & $\begin{array}{l}\text { Enquête documentaire } \\
\text { Enquête par interview }\end{array}$ \\
\hline 2. & Le traitement et analyse des données & Analyse qualitative \\
\hline 3. & L'interprétation des données & La méthode comparative \\
\hline
\end{tabular}

Les Mythes Du Colonisateur Belge :-

L'objectif premier de cet effort étant le contrôle politique, les Belges tenteront de détecter parmi les tribus celle qui seront le mieux de les seconder. Contrôler un pays surtout si l'on entend pratiquer l'administration indirect ; ne peut se faire sans supplétif. Pour soutenir son effort de civilisation et de domination, le colonisateur forge ainsi de " mythes » qui feront l'objet de nombreuses publications, au point que les intéressés eux-mêmes finiront par y adhérer et s'y identifier, jusqu'à notre époque. Il faut d'ailleurs observer que cette pratique est celle de tous les colonisateurs, qui partout dans le monde s'ingèrent à trouver des alliés locaux dans le pays conquis.

Il semble cependant que, s'agissant des Belges, ce mythe est celui de la supériorité intellectuelle et de l'efficacité. Les Belges estiment que certaines tribus sont plus intelligente que les autres, et donc mieux placées pour servir de relais à leur influence.

Au Congo, deux tribus, les Bangalas et le Baluba seront choisies pour cette raison, tandis qu'au Rwanda et au Burundi ce rôle sera dévolu aux Tutsis. 


\section{La genèse des Conflits Rwandais:-}

Avant la colonisation, le Rwanda constituait depuis plusieurs siècles un Etat Nation, qui avait pleinement intégré ses composantes démographiques. F REYNTJENS le fait remarqué ainsi «depuis le temps immémoriaux, les Rwandais, toutes les ethnies confondues, avaient réussi à cohabiter sur la même terre de leurs ancêtres. Leur habitat entremêlé constitue une preuve de la coxistence non conflictuelle entre les différentes ethnies » ${ }^{1}$.

Les Rwandais se reconnaissaient tous à travers une langue unique et une même culture. Ces bases profondes de l'unité du peuple Rwandais avaient été renforcées par l'occupation d'un même espace géographique ne reconnaissant pas de région pour telle ou telle catégorie sociale ou ethnique.

\section{La genèse des conflits Burundais:-}

Depuis longtemps la majorité de chercheurs placent l'origine de conflit Burundais au début de la période coloniale. En effet, avant la période coloniale tous les Burundais croyaient à un seul Dieu l'Imana, obéissaient à une autorité le Mwami (le Roi) et la justice rendue par le Bashingantahe. A l'échelon national, aucun conflit à caractère ethnique n'a été enregistré à cette époque. Le pouvoir puisait l'essentiel de sa force dans les mœurs et la spiritualité, mais ces valeurs ont beaucoup diminuée avec la colonisation.

\section{La genèse des conflits Congolais:-}

Avant la colonisation les congolais vivaient dans un climat de paix. Apres le départ de colon les conflits ont commencé suite à la caste qu'il avait créée dans la population. Maintenant en 1994, le Génocide Rwandais avait déversé de millions de réfugiés et les militaires, groupes attisés par l'idéologie ethnique. C'est ainsi que les guerres ont commencées en RDC et à tel point que quelques grands penseurs ont qualifié la guerre au Congo étant la première guerre mondiale africaine ${ }^{2}$.

Instruments politiques de gestion des conflits dans la région:-

Cette partie vise à fournir des instruments politiques d'Afrique des Grands Lacs, plus particulièrement le Rwanda, le Burundi et la RD Congo.

\section{Pour le Rwanda:-}

$>$ Les accords de paix d'Arusha de 1993 ;

$>$ La Déclaration du front patriotique Rwandais de 1994 (FPR) ;

$>$ Le Tribunal Pénal international pour le Rwanda (TPIR);

$>$ La JuridictionGacaca ;

$>$ La constitution de 2003.

Cette constitution fait apparaitre quelques nouveautés par rapport aux constitutions précédentes et les réalités spécifiques aux contextes Rwandais post génocide.

Sesprincipaux elements sont:

$>$ Le partage équitable du pouvoir ;

> L'instauration d'un Etat de droit et du régime démocratique pluraliste ;

> La lutte contre l'idéologie génocidaire et toutes ses manifestations ;

$>$ L'éradication des divisions ethniques et régionale ;

$>$ La promotion de l'unité nationale ;

$>$ L'égalité de tous les Rwandais et l'édification d'Etat voué à la justice sociale ;

$>$ Et la recherche permanente de dialogue et de consensus social.

Pour le Burundi:-

$>$ L'Institution de Bashingantahe ;

$>$ La charte de l'unité nationale au Burundi ;

$>$ La négociation de KigobeKajaga de 1994 ;

$>$ La convention de Gouvernement ;

$>$ L'Accord d'Arusha pour la paix et la réconciliation au Burundi de 2000.

${ }^{1}$ F.REYNTJENS, L'Afrique des Grands Lacs en Crise, Paris, Karthala, 1993, p. 93.

${ }^{2}$ MUKANDIRWA, S., Impact de la MONUC dans la résolution des conflits à l'est de la RD Congo, mémoire, Butare, UNR, 2006, p. 2. 
Nous n'allons pas entre en détail de cet accord, mais nous allons relever seulement ses points saillants qui sont :

- $\quad$ Il rejette l'idée de Hutuland et de Tutsiland ;

- $\quad$ Il reconnait le multipartisme comme régime politique au Burundi ;

- Autre disposition importante de cet accord concerne la parité Hutu-Tutsi ;

- $\quad$ Et la formation du gouvernement du G-7 (Pro-Hutu) a 60 et 40\% du G10 (Pro- Tutsi).

II.3 Pour la RD Congo:

- Accord de Lusaka pour le cessez le feu ;

- Accord de paix entre la RD Congo et le Rwanda ;

- Accord Global et Inclusif sur la Transition en RD Congo (signé à Pretoria en 2002).

C'est accord est l'aboutissement du Dialogue Inter Congolais. En fait, les parties au présent accord (le Gouvernement de Kinshasa, le RCD, MLC, l'opposition politique, les forces vives, le RCD/ML, RCD/N et le MaïMaï) voulaient mettre fin aux hostilités qui persistaient dans le pays.

Cet accord est composé de cinq points essentiels, à savoir :

- $\quad$ La cessation des hostilités ;

- Les objectifs de la transition ;

- Les principes de la transition ;

- $\quad$ La durée de la transition et en fin ;

- Les institutions de la transition.

Analyse de la gestion politique des conflits dans la région:-

Notre objectif ici n'est pas seulement de faire une comparaison entre les modèles de gestion, celui du Rwanda, Burundi et du Congo. Mais aussi nous différentierons, les trois modèles de gestion tout en observant le partage du pouvoir sur le plan politique.

\section{Le modèle dans la région des grands lacs:-}

Depuis les années des indépendances, la région des grands lacs connaissait des crises ethno politique. A des temps d'accalmie succédaient des moments des violences extrêmes.

Les années 1959 et 1994 au Rwanda, 1972 et 1993 au Burundi, et 1961, 1996 et 1998 au Congo peuvent être relevées comme correspondants aux temps paroxystique sur lesquels l'opinion internationale a accrochée son intention.

Par nature et compte tenu de la composition sociologique et anthropologique politiquement instrumentalisée dans ces pays, un phénomène de contagion s'est développé de part et d'autre de la frontière.

A part des dimensions régionales, les conflits nationaux ont conféré à l'application "Afrique des Grands lacs" un véritable sens géographique qui a déterminé la Communauté Internationale à élaborer des instruments de règlement des conflits. Ces instruments diffèrent d'un pays en un autre. Ainsi par exemple au Rwanda, la disqualification du régime génocidaire a permis l'émergence d'un nouveau leadership qui a su inventer un modèle national, modèle fondé sur la nation, qui est un principe. Le Burundi, qui a connu presque les mêmes problèmes a choisi le modèle de la représentation ethnique, donc un modèle qui institutionnalise l'ethnie. Par contre la RD Congo a institutionnalisé les parties politiques.

\section{Modèle Rwandais:-}

Avant la colonisation le Rwanda était une monarchie, le Roi était à la tête du pays. Même si le phénomène d'inégalité existait entre les ethnies, les Hutus, les Tutsis et les Twa furent toujours valoir leur droit à se substituer au Mwami.

La colonisation Allemande, puis Belge et l'éducation était prise en charge par l'église catholique (les pères blancs) durcirent les oppositions en les formalisant. Les colonisateurs s'appuyant sur une élite, le concept de noblesse et pasteur étaient attribué à la désignation de tutsi. Ils avantagèrent le statut d'éleveur, et, en même temps, favorisèrent l'accès de la noblesse à un pouvoir et un savoir de type occidental. Peu à peu, le non noble et non éleveur majoritaires se trouvent cantonner dans une sorte de pauvreté économique culturelle et symbolique qui vit 
progressivement figurant racial. L'introduction des cartes d'identité, ethnique par la colonisation Belge figea la situation ${ }^{3}$.

A la fin des années cinquante, au tournant de décolonisation les élites Tutsis affichèrent une volonté d'émancipation politique contre la couronne Belge et idéologique contre l'omniprésence de l'église renversèrent alors leurs alliances et soutiennent un processus de démocratisation à vocation majoritaire (six Hutu pour un Tutsi).

Ce renversement représente comme la révolution d'un tiers contre l'aristocratie étant voie la libération contre un occupant de race étrangère suscita des épisodes très violents.

Apres l'indépendance, s'instaure le régime discriminatoire qui n'hésite pas à commettre des massacres et du génocide. L'idéologie anti tutsi et le régionalisme ont été remarqués dans cette période.

Le Président Kayibanda installa son régime sur la terreur anti Tutsi. Une propagande intense, omniprésente occupait les discours des dirigeant, les ondes de la radio, des chansons populaires dans les salles de classe. Cette propagande expliquer que le Tutsi est un étranger qui avait conquis et mis sous servage quatre siècles le people Hutu, que la révolution et la République étaient l'expression de la victoire de peuple majoritaire Hutu sur la majorité féodale Tutsi. L'ostracisme et l'intimidation des Tutsi par le régime Kayibanda furent suivie de l'exclusion graduelle de ses alliés politique et de leurs bases sociales.

Le régime de Habyarimana avait aussi les politiques, discriminatoire ethnique et régionale sur le plan politique et social, il instaura la politique d'équilibre ethnique et régional. Les Tutsis sont exclus des postes politiques et des fonctions administratives et de l'armée, le Hutu du centre et du sud, furent aussi évincé de leur position dominante.

Après les événements tragiques de 1994, les Rwandais se sentaient séparés, ils ont besoin de vivre ensemble afin de construire leur pays dans la paix et la sécurité. Pour y arriver, il faut une nation solide, une nation sans caste, sans ethnie. C'est dans cette perspective que le Rwanda a choisi la voie de la citoyenneté construite après préalables du droit, de l'égalité et du refus de l'institutionnalisation de l'ethnicité.

Cette orientation présente plusieurs avantages politiques dont le plus important est d'éduquer les gouvernants et les gouvernés à mettre en avant le mérite et l'identité citoyenne.

\section{Le modèle Burundais:-}

Avant l'arrivée des colonisateurs, le Burundi était une monarchie, tous les Burundais obéissaient à une seule autorité Umwami (Roi) et la justice était d'une façon équitable au nom du peuple Burundais.

Apres la deuxième guerre mondiale, les Belges cherchaient à gouverner par l'entremise de la monarchie, mais leur politique ont servi à éliminer la complexité et la flexibilité des systèmes socio politique coloniale et a classé les Hutus, les Tutsis et le Twa dans une catégorie ethnique. Appliquant leurs propres théories racistes de la hiérarchie entre le peuple. La politique de discrimination et régionalisme ont été aussi au Burundi.

Dans les années qui ont suivi l'indépendance du Burundi, concédée par la Belgique, son dirigeant, Mwambusta, a cherché à éviter une révolution similaire en équilibrant les intérêts Tutsi et Hutu. Mais alors que les conflits entre les groupes se multipliaient, il s'est de plus en plus associé aux intérêts Tutsis et a perdu son rôle d'arbitre.

Le nouveau Roi n'a pas réussi à imposer son intronisation, et les officiers militaires Tutsis ont nommé Michel Micombero à la présidence. En 1972, beaucoup des Hutus ont été exécutés, c'est ce que le marchand a qualifié comme " génocide sélectif » ${ }^{4}$, l'objectif était d'éliminé effectivement toutes éventuelles menaces Hutue à l'hégémonie Tutsi.

Au cours de vingt années qui ont suivi les massacres de 1972, les Hutus ont été presque exclu de la fonction politique, de l'armée, des écoles et d'autres postes, même sous le régime de Bagaza. Il a également lancé une campagne contre l'église catholique et protestant, et expulsant car ils le soupçonnaient de radicaliser les Hutus.

\footnotetext{
${ }^{3}$ FRANCOIS, X., La complicité de génocide, Ed. La découverte Paris, 1994, p. 21.

${ }^{4}$ LE MARCHAND et DAVID MARTIN, le Génocide sélectif au Burundi, Londres: Minority Right group, 1973, pp.18-19.
} 
Pour mettre fin à cette situation conflictuelle, un accord de paix a été signé à Arusha en 2000. Depuis ce jour, une philosophie de partage du pouvoir sur base ethnique s'est installée.

Le modèle Burundais est un modèle de la construction de la citoyenneté. Il a une attitude d'un modèle Anglo-Saxon. La nation voit sa reconnaissance formelle dans des ethnies représentées, dans les partis politiques regroupés comme on l'observe au Burundi ; elle se voit dans ce qu'on appelle G10 (Tutsi) et G7 (Hutu) ${ }^{5}$. Il est connu sous le nom d'un prétendu "réalisme politique"; les acteurs politiques adoptent donc la reconnaissance formelle des ethnies. Ce modèle prépare le terrain d'une culture de compromis même s'il fait courir un danger d'ordre ethnique à la société.

Ce modèle, en fait, différent totalement du modèle Rwandais, alors que ce dernier met avant la construction de la société et de l'Etat sur l'identité nationale.

\section{Le Modèle Congolais:-}

Le modèle Congolais se différencie de deux autres modèles cités précédemment. Dans cette partie nous parlerons sur l'Accord Global Inclusif sur la transition en RD Congo. C'est accord est l'aboutissement du Dialogue Inter Congolais. En fait, les parties au présent accord (le Gouvernement de Kinshasa, le RCD, MLC, l'opposition politique, les forces vives, le RCD/ML, RCD/N et le MaïMaï) voulaient mettre fin aux hostilités qui persistaient dans le pays. C'est ainsi que, lors de ces accords ils se sont partagé le pouvoir selon le quota de partis politiques. La RD Congo considère le multipartisme, comme l'un des fondements de la démocratie. En effet, il est fondamental que tous les points de vue puissent s'exprimer librement, et que plusieurs partis politiques d'opinions différentes puissent s'affronter pour l'intérêt de toute la nation.

Le modèle Congolais est totalement différent des autres. Malgré certaines similitudes entre les crises, chaque pays privilégie une approche qui traduit, pour le Rwanda, l'aspiration à une nation qui transcende l'ethnisme, pour le Burundi, une nation ou les composantes ethniques sont formellement reconnue et pour la Congo une nation ou les partis politiques jouent un rôle important dans la gestion des affaires publiques (respublica).

\section{Les effets de la guerre dans la région:-}

Les pays qui vivent dans la paix mais ont pour voisin un pays en proie à la guerre en subissent les conséquences à la fois immédiates et à long terme. Pour notre cas, nous allons retenir que deux, qui sont les suivantes :

\section{Conséquences économiques:-}

Les guerres ne sont pas dommageables pour les pays qui en sont frappés. Les pays voisins voient déferler chez eux de nombreux réfugiés; les victimes de la guerre ont en effet rarement les moyens de subvenir à leur besoin. En titre exemplatif, de million de réfugiés Rwandais que le Congo a du accueillir sur son territoire lors du génocide des Tutsis au Rwanda, représentait pour le Congo une charge considérable. Cet afflux de réfugiés a des conséquences importantes au niveau régional. D'autres effets se font sentir : alourdissement du budget militaire, augmentation du coût des transports et détérioration de l'image de la région aux yeux des investisseurs étrangers.

\section{Conséquences sociales:-}

Les effets le plus immédiat de la guerre sur les pays voisins est l'arrivée massive sur le territoire de millier de réfugiés avec tous les inconvénients que cela entraîne, pour la population locale. Dans la mesure où les réfugiés restent dans les pays qui leur ont donné l'asile longtemps après la fin de la guerre, les effets sociaux de guerre sur ces pays d'accueil sont aussi durables. La guerre fait de nombreuses victimes dans les pays voisins par propagation des maladies, notamment le paludisme, cholera, etc.

L'afflux massif de réfugiés entraîne aussi leur entassement dans les camps surpeuplés ou ils n'ont ni accès à l'eau potable ni à une nourriture saine, c'est là crée un environnement idéal pour la propagation des maladies infectieuses.

La paix dans la région des grands lacs:-

Si l'on veut établir la paix dans nos pays, il faut que tout le peuple soit invité à participer à sa construction. Est-ce que le peuple est-il préparé ? Oui se soucie de l'enseigner ? Sans doute dans les grandes villes, la population a accès

\footnotetext{
${ }^{5}$ SHYAKA, A., Les conflits en Afrique des Grands Lacs et Esquisse de leur Résolution, Ed. Académique, Dialog, Varsovie, 2003, p. 212.
} 
aux journaux, à la télévision, etc. Mais que pensons-nous de million de villageois que regorge notre région qui ont aussi droit aux enseignements?

Une population non préparée est à la merci des démagogues. La paix n'est pas un cadeau, mais au contraire le fruit des efforts de toute la population. On ne fait pas un médecin en donnant un diplôme de médicine à un analphabète, ce serait un mensonge.

Sans sérieuse préparation, les meilleurs projets de la société échouent. Il faut donc informer et former la population sur la cohabitation pacifique.

Mais ne nous faisons pas d'illusion, une brochure, même si elle répond à un réel besoin, est totalement inefficace par elle-même. Il faut que, les apôtres de la paix prennent les initiatives de l'étudier et de l'expliquer clairement dans les milieux qu'ils peuvent atteindre.

Ces apôtres de la paix, ce sont les nombreuses ONG crées pour la protection des droits humains, ce sont aussi les groupes informels, les associations de toutes sortes et les églises. Elles ont pour mission essentielle d'établir entre les hommes une attente fraternelle. Cette entente dépendra en grande partie de la bonne organisation du pays sur une base vraiment démocratique.

\section{Education du peuple à la démocratie:-}

Beaucoup de ceux qui revendiquent la démocratie confondent celle-ci avec l'anarchie. Ils s'imaginent qu'ils deviendront libres par la force. Or le refus d'une loi qui s'impose à tous, est le plus grand obstacle à l'instauration d'une véritable démocratie.

Pour que la démocratie prenne racine, il est nécessaire que chacun accepte de s'imposer une discipline. Il est donc nécessaire qu'il y ait des lois et que ces lois soient respectées par tous.

Dès l'antiquité, on avait remarqué que l'anarchie, impunité, absence des lois, conduisait toujours à la dictature. L'anarchie crée l'insécurité, l'insécurité provoque la peur.

Tous nous voulons la démocratie après les décennies de régime autoritaire et des partis uniques. Nous devons accepter de nous discipliner pour vivre dans la Région des Grands Lacs en paix avec comme principe vivons " l'unité dans la diversité ».

\section{Conclusion:-}

Notre travail a porté sur la gestion politique des conflits dans la Région des Grands Lacs (Rwanda, Burundi, et Congo). Dans ce travail, il a été question de savoir si la reconstruction de l'identité rwandaise est l'un des moyens d'atteindre l'unité et la réconciliation des Rwandais, d'autre part à celle de savoir si la reconstruction de la Nation Burundaise serait l'institutionnalisation de l'ethnicité et au Congo de savoir si la reconstruction de la Nation Congolaise serait l'un des moyens de résoudre les conflits par le partage de pouvoir par les partis politiques.

Il est assez logique pour l'étude des conflits dans la région que nous poussions traiter leurs causes et leurs conséquences. En effet, les conflits dans nos trois pays d'étude ont trouvé leurs origines dans la période coloniale. Les colonisateurs ont joué un rôle très important dans la stratification des ethnies et dans la division des peuples de ces pays. En ce qui concerne les conséquences de ces conflits au Rwanda (le génocide de 1994), au Burundi (la crise de 1993) et en RD Congo (la guerre de 1998).

Les instruments politiques que le Rwanda a mis en place dans le but de reconstruire l'unité et la réconciliation des Rwandais. Ces instruments s'inspirent de la reconstruction d'une nation Rwandaise, la Rwandité.

Les instruments politiques que les Accords d'Arusha sur la paix et la réconciliation ont prévus afin de résoudre le conflit Burundais. Cet instrument c'est institutionnalisation de l'ethnie (quota ethnique).

Les instruments politiques que l'accord global et inclusif sur la transition en Congo, s'inspire de la reconstruction de la nation par le partage du pouvoir entre les partis politiques (quota des partis politiques). 
Apres une étude minutieuse sur ces trois modèles de résolution des conflits, nous pouvons confirmer que le modèle Rwandais peut être le meilleur, car il ne divise pas la population, mais le problème peut se pose une fois qu'on se fait des illusions. Donc, on ne peut affirmer totalement que ce modèle est parfait car l'homme change tout le temps. En outre, on ne peut même pas honorer le problème à base identitaire qui, jusqu'ici, cause des sérieux problèmes dans notre région. En fait, ce qui est nécessaire, c'est de renforcer des relations pour construire la citoyenneté qui est un élément recherché pour la reconstruction de notre sous-région.

\section{Recommandations:-}

1. Développer des mécanismes communautaires de réponse rapide basés sur un suivi des dynamiques de conflit et sur l'élaboration de piste d'action opérationnelles en mettant l'accent sur le rôle et responsabilité des acteurs locaux ;

2. Exploiter toutes les potentialités des réseaux universitaires de la région, notamment en vue d'une production scientifique davantage objective, susceptible d'apporter sa contribution spécifique à une paix durable ;

3. Prendre en compte des revendications des appartenances ethniques vivant de part et d'autre de la frontière.

\section{Bibliographie:-}

Ouvrages :-

1. FRANCOIS, X., La complicité de génocide, Ed. la découverte Paris, 1994.

2. F.REYNTJENS, L'Afrique des Grands Lacs en Crise, Paris, Karthala, 1993

3. LE MARCHAND et DAVID MARTIN, le Génocide sélectif au Burundi, Londres:

4. Minority Right group, 1973.

5. SHYAKA, A., Les conflits en Afrique des Grands Lacs et Esquisse de leur Résolution,

6. Ed. Académique, Dialog, Varsovie, 2003.

\section{Reference Electroniques :-}

1. http:// afriquepluriel.ruwenzori.net/atlas-total.htm

2. www.google.fr

\section{Memoires:-}

MUKANDIRWA, S., Impact de la MONUC dans la résolution des conflits à l'est de la RD Congo, mémoire, Butare, Université Nationale Rwanda, 2006. 